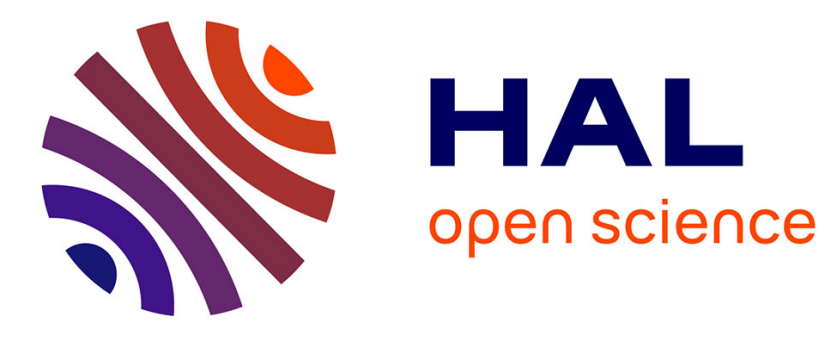

\title{
Direction finding antenna arrays for the randomly located source
}

Houcem Gazzah, Jean-Pierre Delmas

\section{To cite this version:}

Houcem Gazzah, Jean-Pierre Delmas. Direction finding antenna arrays for the randomly located source. IEEE Transactions on Signal Processing, 2012, 60 (11), pp.6063-6068. 10.1109/TSP.2012.2211592 . hal-00746440

\section{HAL Id: hal-00746440 \\ https://hal.science/hal-00746440}

Submitted on 29 Oct 2012

HAL is a multi-disciplinary open access archive for the deposit and dissemination of scientific research documents, whether they are published or not. The documents may come from teaching and research institutions in France or abroad, or from public or private research centers.
L'archive ouverte pluridisciplinaire HAL, est destinée au dépôt et à la diffusion de documents scientifiques de niveau recherche, publiés ou non, émanant des établissements d'enseignement et de recherche français ou étrangers, des laboratoires publics ou privés. 


\title{
Direction Finding Antenna Arrays for the Randomly Located Source
}

\author{
Houcem Gazzah and Jean-Pierre Delmas
}

\begin{abstract}
We consider the problem of sensor placement for estimating the direction of arrival of a narrow-band source randomly located in the far-field of a planar antenna array. Performance is evaluated by means of the expectation of the conditional Cramer Rao bound, normalized to that of the uniform circular array. Two cost functions are obtained, relative to azimuth and elevation, respectively. They depend on the array geometry as well as the distribution of the source azimuth. A class of uniform antenna arrays is investigated. It is adapted to the particular probabilistic distribution of the azimuth, while ensuring protection against array ambiguities. Using an exhaustive search procedure, we either seek the same reduction of both cost functions, or rather focus on one in particular. In the first approach, we achieve a reduction of almost $36 \%$ of both, regardless of the source azimuth distribution. In the second approach, we can obtain larger reductions for the targeted parameter. In both cases, optimal arrays are close to the $\mathrm{V}$ shape, for which performance analysis is conducted and closed-form expressions are obtained.
\end{abstract}

\section{INTRODUCTION}

Direction of Arrival (DOA) is a topic of the utmost importance in statistical signal processing. A set of sensors collect signal snapshots to estimate the source DOA. Estimation accuracy depends on the sensor positions, in a way that has remained largely unquantified [1], [2], mainly because of the complexity of the Cramer Rao Bound (CRB), even in the single source case [3]. Because of the intricate original expression of the CRB [3], early attempts to achieve array optimization were conducted mostly using heuristic techniques [4], [5]. A recent simplification of the CRB of the planar antenna array shows a convenient sinusoidal dependency on the source azimuth [6], as long as the array geometry is concerned. Other parameters are eliminated by normalizing to the uniform circular array (UCA).

Houcem Gazzah is with the Department of Electrical and Computer Engineering, University of Sharjah, 27272, UAE. Email: hgazzah@sharjah.ac.ae, Tel.: (971) 6.5050.917, Fax.: (971) 6.5050.872. Jean-Pierre Delmas is with Telecom SudParis, Departement CITI, CNRS UMR 5157, Evry, France. E-mail: jean-pierre.delmas@it-sudparis.eu. 
Estimation accuracy increases if sensors are allowed to occupy a larger region [4], at the expense of increased array ambiguities [7]. They occur when two (first-order ambiguity) or more (higher-order ambiguity) steering vectors at different look directions are linearly dependent [8]. A robust manner to avoid ambiguities is to impose a regular (half-wavelength) spacing between adjacent sensors, because, then, the matrix of steering vectors is less likely to be close to singularity [7]. Global optimization of the array geometry, now function of a set of angular parameters, can be achieved by exhaustive search, an approach followed in [9], [10] to design optimal arrays for a source with a fixed DOA. The main outcome was a set of optimal isotropic arrays whose CRB for azimuth and elevation, just like for the UCA, do not depend on the actual source azimuth. However, they are $29 \%$ lower than their UCA counterparts.

In wireless cellular systems, for example, the DOA distribution around the base station is sought after in order to improve the network design, performance, and services [11]. The incorporation of such a knowledge into the array optimization procedure is expected to further improve DOA estimation accuracy. The application of the theory of Bayesian estimation to this situation leads to the expected CRB (ECRB) as a performance measure. Normalization to the ECRB of the UCA results in two cost functions (relative to the azimuth and elevation angles) that depend only on the array geometry and on the Probability Density Function (PDF) of the source azimuth. By taking the DOA distribution into consideration, we obtain arrays with better estimation capabilities, but we also prove that optimal (minimizing the CRB) isotropic arrays are not optimal (i.e., do not minimize the ECRB) even in the case of a uniformly distributed DOA, contrary to claims in $[4$, Sec. V] and $[12$, Sec. V].

Two optimization approaches are proposed, depending on whether the two DOA angles are treated equally, or whether one is prioritized over the other. In the first approach, numerical examples show a reduction, w.r.t. the UCA, of the ECRB of $36 \%$ on both azimuth and elevation angles. This performance is achieved by arrays that are not isotropic, an improvement w.r.t. [9], [10] where optimal isotropic arrays achieve a $29 \%$ reduction. In the second approach, the emphasis is put on one DOA angle in particular (the azimuth angle for example) and the associated ECRB can be further reduced.

The exhaustive search procedure is computation-consuming for large array sizes. Also, optimal arrays have systematically shown a V-like structure. V arrays have already been shown to be near-optimum for CRB-based geometry optimization [9], [10]. This motivates a detailed analysis of the performance of V arrays and their subsequent optimization. Interestingly, for large-sized V arrays, the normalized (w.r.t. the UCA) ECRB is shown to be independent of the array size. Analytical expressions are obtained that determine the shape, the orientation and the DOA estimation accuracy of the optimal V array in the presence of a source with a known arbitrary azimuth distribution. 
We denote by E [.] the expectation operator. In Sec. II, we introduce the observation model and recall previous results. In Sec. III, we develop a Bayesian CRB based criterion and apply it to uniform arrays. Sec. IV presents a detailed study of V arrays. Finally, a conclusion is given in Sec. V.

\section{Data Model And Performance Criteria}

We consider an antenna array made of $M$ identical and omni-directional sensors in the $(x, y)$ plane. The position of the $m$-th sensor is given by $\gamma_{m} \hat{=} \rho_{m} \exp \left(j \phi_{m}\right)$ where $\rho_{m}$ is the distance to the origin and $\phi_{m}$ is the angle to $[O, x)$. A far-field source is emitting a narrow-band signal $s(t)$ with wavelength $\lambda$. The DOA is specified by azimuth $\Phi$ and elevation $\Theta$, so that the snapshot collected by sensor $m$ at time index $t$ is $x_{m}(t)=\exp \left[2 j \pi \frac{\rho_{m}}{\lambda} \sin (\Theta) \cos \left(\Phi-\phi_{m}\right)\right] s(t)+n_{m}(t)$, where $n_{m}(t)$ represents the ambient additive noise collected by sensor $m$. Snapshots $\mathbf{x}\left(t_{1}\right), \cdots, \mathbf{x}\left(t_{N}\right)$, where $\mathbf{x}(t) \hat{=}\left[x_{1}(t) \cdots x_{M}(t)\right]^{T}$, are used to estimate the source DOA, i.e., parameters $\Phi$ and $\Theta$, using a variety of algorithms.

Under the assumptions of (i) circular, Gaussian, zero-mean and mutually independent source and noise signals with respective power $\sigma_{s}^{2}$ and $\sigma_{n}^{2}$; (ii) independent and identically distributed source snapshots and (iii) noise samples collected from different sensors and/or at different time indexes are mutually independent; the (concentrated) CRB [3, rel.(39)] has been greatly simplified (under no additional assumptions) [6] to obtain, in particular, $C_{\Phi \Phi}=\frac{A}{\sin ^{2}(\Theta)} B(\Phi)$ and $C_{\Theta \Theta}=\frac{A}{\cos ^{2}(\Theta)} B\left(\Phi+\frac{\pi}{2}\right)$, where $A$ is a constant that depends on the array size, the observation SNR and the number of snapshots. Only $B(\Phi) \hat{=}\left\{S_{0}+\Re\left[S_{1} \exp (-2 j \Phi)\right]\right\} /\left(S_{0}^{2}-\left|S_{1}\right|^{2}\right)$ depends on the array geometry through

$$
\begin{aligned}
& S_{0} \hat{=} \sum_{m=1}^{M}\left|\frac{\gamma_{m}}{\lambda}\right|^{2}-\frac{1}{M}\left|\sum_{m=1}^{M} \frac{\gamma_{m}}{\lambda}\right|^{2}, \\
& S_{1} \hat{=} \sum_{m=1}^{M}\left(\frac{\gamma_{m}}{\lambda}\right)^{2}-\frac{1}{M}\left(\sum_{m=1}^{M} \frac{\gamma_{m}}{\lambda}\right)^{2} .
\end{aligned}
$$

This simple sinusoidal form allows one to analyze the impact of the array geometry on DOA estimation, and, ultimately, to optimize such a geometry [9]. Of a particular interest are arrays (like the UCA for example) for which $S_{1}$ is zero and, as a consequence, $B(\Phi)$ is a constant. We refer to them as isotropic arrays because, for a given elevation angle, the CRBs on azimuth and elevation do not depend on the actual azimuth angle. Non-trivial optimal isotropic antenna arrays obtained in [10] achieve a CRB that is $29 \%$ (for large-sized arrays) lower than that of the UCA, and so for both azimuth and elevation angles. In this paper, we do not require arrays to be isotropic and, so, $S_{1}$ is not necessarily equal to zero.

In practice, there are situations where a priori knowledge is available about the DOA parameters with $(\Phi, \Theta)$ following some known joint distribution. We adopt a Bayesian approach by using [13, 
p. 6] the expectation of the conditional CRBs (ECRB) $\bar{C}_{\Phi \Phi} \hat{=} \mathrm{E}_{\Phi, \Theta}\left(C_{\Phi \Phi}\right)$ and $\bar{C}_{\Theta, \Theta} \hat{=} \mathrm{E}_{\Phi, \Theta}\left(C_{\Theta \Theta}\right)$ as criteria to minimize. The ECRB was also used in [4] as a cost function, but only the azimuth DOA angle was considered therein. Advantageously, these cost functions inherit the convenient structure of the CRB. If we assume the azimuth and elevation angles to be independently distributed, we have $\bar{C}_{\Phi \Phi}=A \mathrm{E}\left[\frac{1}{\sin ^{2}(\Theta)}\right] \mathrm{E}[B(\Phi)]$ and $\bar{C}_{\Theta, \Theta}=A \mathrm{E}\left[\frac{1}{\cos ^{2}(\Theta)}\right] \mathrm{E}\left[B\left(\Phi+\frac{\pi}{2}\right)\right]$.

Because the above expressions have separate terms in $\Phi$ and $\Theta$, where only the $\Phi$-dependent term is function of the array geometry, the use of a reference antenna renders the criterion (and any subsequent optimization) independent of the source elevation. The UCA, our reference antenna, has $M$ sensors spaced by $d$ and verifies $B_{\mathrm{UCA}} \hat{=} B(\Phi)=4 \lambda^{2} \sin ^{2}(\pi / M) /\left(M d^{2}\right)$. If we denote by $\varphi_{\Phi}(x) \hat{=} \mathrm{E}[\exp (j \Phi x)]$ the characteristic function relative to the random parameter $\Phi$, we straightforwardly obtain

$$
\begin{aligned}
\frac{\bar{C}_{\Phi \Phi}}{\left.\bar{C}_{\Phi \Phi}\right|_{\mathrm{UCA}}} & =\frac{1}{B_{\mathrm{UCA}}} \frac{S_{0}+\Re\left[S_{1} \varphi_{\Phi}(-2)\right]}{S_{0}^{2}-\left|S_{1}\right|^{2}} \\
\frac{\bar{C}_{\Theta \Theta}}{\left.\bar{C}_{\Theta \Theta}\right|_{\mathrm{UCA}}} & =\frac{1}{B_{\mathrm{UCA}}} \frac{S_{0}-\Re\left[S_{1} \varphi_{\Phi}(-2)\right]}{S_{0}^{2}-\left|S_{1}\right|^{2}} .
\end{aligned}
$$

Obviously, in the case of a randomly (not only uniformly, as assumed in [14]) distributed azimuth angle, an isotropic array (one for which $S_{1}$ equals zero) exhibits the same normalized ECRB for azimuth and elevation that, furthermore, does not depend on the source probabilistic distribution. However, the opposite is not true: The normalized ECRBs can be independent of $\Phi$ without the array being necessarily isotropic. In fact, the normalized ECRBs do not depend on the azimuth if

$$
\Re\left[S_{1} \varphi_{\Phi}(-2)\right]=0 .
$$

Except when the array is isotropic (i.e., $S_{1}=0$ ), we can rewrite the above as

$$
\mathrm{E}[\cos (2 \Phi)] \cos \left[\arg \left(S_{1}\right)\right]+\mathrm{E}[\sin (2 \Phi)] \sin \left[\arg \left(S_{1}\right)\right]=0
$$

This relationship between the probabilistic distribution of the azimuth and the array geometry ensures that the two normalized ECRBs are identical, reducing (3) and (4) to a unique performance measure

$$
\bar{C}=\frac{1}{B_{\mathrm{UCA}}} \frac{S_{0}}{S_{0}^{2}-\left|S_{1}\right|^{2}}
$$

Condition (5) is satisfied when the antenna is isotropic and/or when the distribution is uniform over $[-\pi, \pi)$, but also if $\varphi_{\Phi}(-2)=\mathrm{E}[\exp (2 j \Phi)]=0$, which is not only the case of the uniform distribution.

\section{OPTIMIZATION OF UNIFORM ARRAYS}

The design of antenna arrays is made difficult by the requirement that the array be ambiguity-free. Formally, rank ambiguity occurs when steering vectors corresponding to different look directions are 
linearly dependent [8], and a number of sufficient conditions have been developed to prevent this from taking place. We have chosen to implement the weakest condition [8, Th. 9] because it only involves spacings between sensors, while other conditions involve orientation (angular) parameters as well [15], [16], [17]. The condition [8, Th. 9] applies to a set of three arbitrarily chosen sensors. It ensures that two steering vectors (associated with two different look directions) are not colinear. However, this does not prevent them from nearly being so. In fact, the definition of ambiguity is sometimes extended to include cases where the matrix of steering vectors is close to singular [18]. This is particularly severe for non uniform arrays, for which the inter-sensor spacing is not constant [7]. The problem is intractable even in the simplest case of linear arrays [18]. By imposing a constant inter-sensors spacing, our approach is sub-optimal in the strict sense, but is robust from the ambiguity point-of-view. It is also interesting from the analytical and computational points-of-view, as we obtain expressions in terms of bounded parameters, which are amenable to global optimization by systematic search.

We assume sensors to be placed at a constant spacing $d$ along a curve originating at the origin, following the relationship $\gamma_{m}=\gamma_{m-1}+d \exp \left(j \psi_{m-1}\right)$. The normalized sensor coordinates $\gamma_{m} / d$ are function only of the $M-1$ angular parameters $\psi_{1}, \cdots, \psi_{M-1}$, each in $[-\pi, \pi)$. Thanks to this structure, the optimization problem is rendered independent of the actual spacing $d$. In fact, if we introduce the following $d$-independent parameters that describe the array geometry: $T_{0} \hat{=} \frac{\lambda^{2}}{d^{2}} S_{0}=\sum_{m=1}^{M} \frac{\left|\gamma_{m}\right|^{2}}{d^{2}}-\frac{1}{M}\left|\sum_{m=1}^{M} \frac{\gamma_{m}}{d}\right|^{2}$ and $T_{1} \hat{=} \frac{\lambda^{2}}{d^{2}} S_{1}=\sum_{m=1}^{M} \frac{\gamma_{m}^{2}}{d^{2}}-\frac{1}{M}\left(\sum_{m=1}^{M} \frac{\gamma_{m}}{d}\right)^{2}$, then, (3) and (4) can be updated as follows

$$
\begin{aligned}
\frac{\bar{C}_{\Phi \Phi}}{\left.\bar{C}_{\Phi \Phi}\right|_{\mathrm{UCA}}} & =\frac{1}{\mathcal{B}} \frac{T_{0}+\Re\left[T_{1} \varphi_{\Phi}(-2)\right]}{T_{0}^{2}-\left|T_{1}\right|^{2}} \\
\frac{\bar{C}_{\Theta \Theta}}{\left.\bar{C}_{\Theta \Theta}\right|_{\mathrm{UCA}}} & =\frac{1}{\mathcal{B}} \frac{T_{0}-\Re\left[T_{1} \varphi_{\Phi}(-2)\right]}{T_{0}^{2}-\left|T_{1}\right|^{2}}
\end{aligned}
$$

where $\mathcal{B} \hat{=} 4 \sin ^{2}(\pi / M) / M$ does not depend on $d$ neither. The above (8) and (9) form two cost functions that depend on the probabilistic distribution of the azimuth via $\varphi_{\Phi}(-2)$ and on the array geometry via $T_{0}$ and $T_{1}$, i.e. via $\psi_{1}, \cdots, \psi_{M-1}$.

\section{A. Unprioritized Approach}

By unprioritized, we mean that both azimuth and elevation are of equal importance and the same level of enhancement (w.r.t. the UCA) is required, i.e. (8) and (9) should be the same. This yields to the condition (5) and that (8) and (9) reduce to (7). On one hand, we minimize (7), which is a function of $S_{0}$ and $\left|S_{1}\right|$, that remain unchanged if the antenna array is rotated. On the other hand, and independently from (7), we have to respect the constraint (6), which is a function of the argument of $S_{1}$. In fact, 
minimizing (7) and then using the constraint in (6) for fixing the orientation gives the same solution obtained from minimizing (3) and (4) under the constraint in (6). Optimizing the cost function (7) helps determine the shape of the optimal array, while solving the constraint (6) helps fix its orientation. These two independent steps are detailed now.

The minimization of the cost function $\bar{C}$ in (7) is equivalent to maximizing

$$
T_{0}-\frac{\left|T_{1}\right|^{2}}{T_{0}} .
$$

This is to be compared to optimal isotropic arrays obtained in [10] where the above was maximized under the constraint of $T_{1}$ equal to zero. By relaxing this constraint, we are able to obtain non-isotropic arrays that outperform any isotropic array from the Bayesian point-of-view, i.e., in terms of the ECRB.

Notice that (i) arrays that are obtained one from the other by axis-symmetry w.r.t. the $x$-axis and (ii) arrays that are rotated versions of each other, have identical $T_{0}$ and $\left|T_{1}\right|$. Hence, the second sensor can be fixed with $x=d$ (i.e., $\psi_{1}=0$ ) while the third sensor can be constrained to be in the positive $y$ semi-plane (i.e., positive $\psi_{2}$ ). For $m>2, \psi_{m}$ spans the interval $[-\pi, \pi$ ). If we adopt a step $d \psi$ (fixed to $d \psi=\pi / 50$ in our calculations), the so-implemented exhaustive grid-search involves testing $2^{M-3}(\pi / d \psi)^{M-2}$ candidate tuples $\psi_{2}, \cdots, \psi_{M-1}$, among which we pick the tuple with the largest $T_{0}-\left|T_{1}\right|^{2} / T_{0}$.

\begin{tabular}{|r||c|c|c|c|c||c|c|c|c|}
\hline Normalized ECRB $\bar{C}$ & $M=4$ & $M=5$ & $M=6$ & $M=7$ & $M=8$ & $M=9$ & $M=10$ & $M=11$ & $M=12$ \\
\hline Optimal array & 1 & 0.7306 & 0.7039 & 0.6805 & 0.6689 & 0.6597 & 0.6540 & 0.6494 & 0.6461 \\
\hline Optimal isotropic array & 1 & 0.8075 & 0.8005 & 0.7617 & 0.7566 & 0.7441 & 0.7398 & 0.7331 & 0.7302 \\
\hline
\end{tabular}

TABLE I

NORMALIZED ECRBS OF OPTIMAL ARRAYS [MAXIMIZING $T_{0}-\left|T_{1}\right|^{2} / T_{0}$, I.E. (10)] AND OF OPTIMAL ISOTROPIC ARRAYS (MAXIMIZING $T_{0}$ UNDER $T_{1}=0[9]$ ).

Optimal arrays obtained for $M$ less than or equal to 9 exhibit a concave geometry. Hence, in order to reduce the complexity burden (exponential in $M$ ), we impose, for $M$ larger than 9 , that the array has a concave geometry, i.e. $\psi_{m} \geq \psi_{m-1}$ for $m \geq 2$. While this significantly limits the search area, it may possibly lead to suboptimal solutions. Except when $M=4$ (the optimal array is the UCA), the so-computed optimal arrays are not isotropic (i.e., they have non-zero $T_{1}$ ). As the number of sensors increases, the ECRB of the optimal array is reduced by $36 \%$ compared to the UCA, and by $10 \%$ compared to optimal isotropic arrays obtained [9], as can be seen in Tab. I. 
For an arbitrary distribution of the azimuth and a predetermined number of sensors $M$, the array obtained using the above procedure has the appropriate shape but needs to be oriented in order to satisfy (6). The normalized ECRB for the optimal array, however, is what is reported in Tab. I. If $T_{1}^{\dagger}$ is relative to the array obtained by maximizing (10) and $T_{1}$ is relative to the optimal array, then we have $T_{1}=T_{1}^{\dagger} e^{2 j \Upsilon}$. Consequently, the rotation is simply given by $\Upsilon=\left[\arg \left(T_{1}\right)-\arg \left(T_{1}^{\dagger}\right)\right] / 2$, where the argument of $T_{1}$ is obtained by solving (6) rewritten as $\mathrm{E}[\cos (2 \Phi)] \cos \left[\arg \left(T_{1}\right)\right]+\mathrm{E}[\sin (2 \Phi)] \sin \left[\arg \left(T_{1}\right)\right]=0$.

\section{B. Prioritized Approach}

In the general case, we have two potentially distinct normalized ECRBs (8) and (9), relative to the azimuth and elevation angles. We suggest the following ad-hoc criterion:

$$
\text { Min } \frac{\bar{C}_{\Phi \Phi}}{\bar{C}_{\Phi \Phi} \mid \mathrm{UCA}} \quad \text { subject to } \quad \bar{C}_{\Theta, \Theta} \leq\left.\bar{C}_{\Theta, \Theta}\right|_{\mathrm{UCA}} \text {. }
$$

where all parameters $\psi_{1}, \cdots, \psi_{M-1}$ are allowed to span the interval $[-\pi, \pi)$. The rationale behind this criterion is that, in many practical situations, the azimuth angle is more important than the elevation angle. The objective here is to find an array that minimizes the MSE on the azimuth angle while not deteriorating, w.r.t. the UCA, the MSE on the elevation angle.

We illustrate this optimization procedure for an azimuth distribution $\sum_{p=1}^{3} \frac{1}{3 \sigma_{p} \sqrt{2 \pi}} \exp \left[-\frac{\left(\Phi-\Omega_{p}\right)^{2}}{2 \sigma_{p}^{2}}\right]$ corresponding to three equally probable look directions at $\Omega_{1}=0^{\circ}, \Omega_{2}=90^{\circ}$ and $\Omega_{3}=200^{\circ}$, known with an uncertainty of $\sigma_{1}=\sigma_{2}=\sigma_{3}=10^{\circ}$. The optimal array is shown in Fig. 1, along with the source azimuth PDF (in polar coordinates). The normalized ECRBs on azimuth and elevation angles [i.e., (8) and (9), respectively] are equal to $67.47 \%$ and $73.31 \%$, respectively. With reference to the UCA, not only do we obtain a significant reduction of the MSE on the azimuth angle, but also a significant reduction of the MSE on the elevation angle, even though this is not the objective of the optimization procedure. In order to compare results with the unprioritized approach, notice that the optimal array [the 6-sensors array in Tab. I, rotated to satisfy (6), i.e., to ensure $\left.\tan \left[\arg \left(S_{1}\right)\right]=-1.1920\right]$ delivers a normalized ECRB equal to $70.39 \%$ (see Tab. 1) for both azimuth and elevation angles.

\section{OptimizATION OF V-ShAPED ARRAYS}

The optimization problems in Sec. III have been solved numerically, but not analytically. While this has been useful to define a procedure to adapt the array geometry to the probabilistic distribution of the azimuth, we remain short of physical insight into this inter-dependency. Interestingly, optimal arrays computed in Sec. III-A (where we have assumed a concave geometry for $M>9$ ) and Sec. III-B show a 


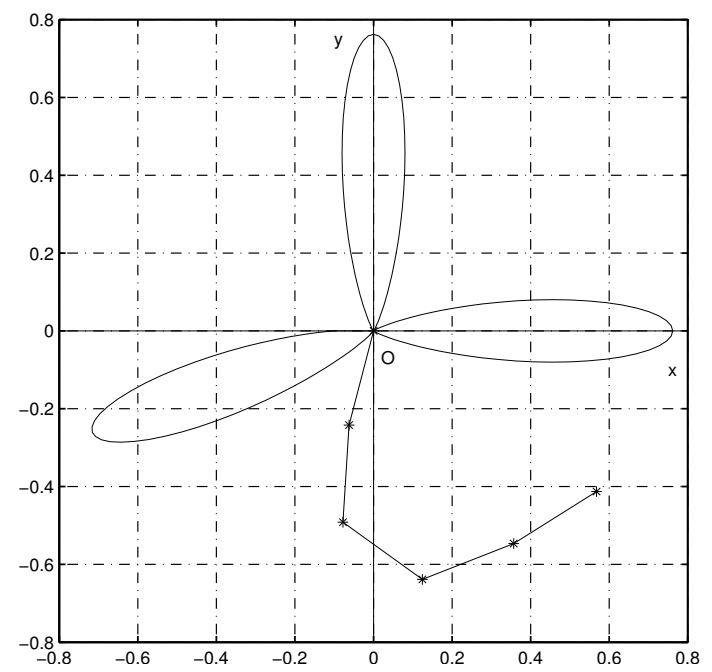

Fig. 1. Optimal placement of 6 sensors (marked by ${ }^{*}$ ' dots) to fit a particular PDF of the azimuth angle (marked by solid line). The curve is a polar representation of the prior distribution of the azimuth angle [For a point $M$ of the curve, distance $O M$ represents the PDF at the azimuth angle $\Phi=[O x, O M)]$.

V-like geometry. This concurs with [10], where V arrays were shown to be near-optimum in estimating the DOA of a fixed source and suggests that performance analysis of $\mathrm{V}$ arrays is meaningful to evaluate the potential of ECRB-optimized arrays. We revisit the developments in Sec. III in the case of a V-shaped array. The $\mathrm{V}$ array geometry is function of only two parameters and, more interestingly, the analysis can be made independent of the array size $M$ when $M$ is large enough.

For simplicity, we assume odd $M$ (even $M$ leads to the same asymptotic expressions [10]). Sensor 1 is placed at the origin. Sensors $2 k$ (resp. $2 k+1), k=1, \cdots,(M-1) / 2$, form a branch at an angle $\Delta_{1}$ (resp. $\Delta_{2}$ ) from the $x$-axis. We assume $\Delta_{1}$ and $\Delta_{2}$ to be both in $[0,2 \pi)$. Following similar steps as in [6], [9], [10], we obtain the following approximations for large $M$

$$
\begin{aligned}
& T_{0} \simeq \frac{5-3 \cos \left(\Delta_{1}-\Delta_{2}\right)}{96} M^{3} \\
& T_{1} \simeq \frac{5 \cos \left(\Delta_{1}-\Delta_{2}\right)-3}{96} \exp \left[j\left(\Delta_{1}+\Delta_{2}\right)\right] M^{3}
\end{aligned}
$$

The above expressions in $M^{3}$ suggest that the analysis for large $M$ may be applicable to practical $\mathrm{V}$ arrays (a comparable analysis in [6, Sec. V] has shown to be accurate for $M$ as low as 5). Notice that for large $M, \mathcal{B} \simeq 4 \pi^{2} / M^{3}$. After tedious manipulations, (8) and (9) are shown to be independent of $M$ :

$$
\frac{\bar{C}_{\Phi \Phi}}{\left.\bar{C}_{\Phi \Phi}\right|_{\mathrm{UCA}}} \simeq \frac{3}{2 \pi^{2}} \frac{5-3 \cos \left(\Delta_{1}-\Delta_{2}\right)+\left[5 \cos \left(\Delta_{1}-\Delta_{2}\right)-3\right] \Re\left[\exp \left[j\left(\Delta_{1}+\Delta_{2}\right)\right] \varphi_{\Phi}(-2)\right]}{\sin ^{2}\left(\Delta_{2}-\Delta_{1}\right)}
$$




$$
\frac{\bar{C}_{\Theta \Theta}}{\left.\bar{C}_{\Theta \Theta}\right|_{\mathrm{UCA}}} \simeq \frac{3}{2 \pi^{2}} \frac{5-3 \cos \left(\Delta_{1}-\Delta_{2}\right)-\left[5 \cos \left(\Delta_{1}-\Delta_{2}\right)-3\right] \Re\left[\exp \left[j\left(\Delta_{1}+\Delta_{2}\right)\right] \varphi_{\Phi}(-2)\right]}{\sin ^{2}\left(\Delta_{2}-\Delta_{1}\right)} .
$$

We can see from the expression of $T_{1}$ in (12) that the $\mathrm{V}$ array is isotropic iff the angle between the two branches is equal to $\Delta_{2}-\Delta_{1}=\arccos (3 / 5)$, i.e. $\Delta_{2}-\Delta_{1}=53.13^{\circ}$, in accordance with [9]. Then, the right-hand side in (13) and (14) becomes equal to $15 /\left(2 \pi^{2}\right)=76 \%$, i.e., a reduction of the ECRB (and also of the CRB) of $24 \%$ compared to the UCA, on both the azimuth and elevation DOA, and so regardless of the actual distribution of the DOA of the randomly located source.

\section{A. Unprioritized Approach}

We now refer to the analysis in Sec. III-A and impose that the normalized ECRBs on the azimuth and elevation are identical. By comparing (13) and (14), this is true when the V antenna is isotropic [i.e., $\left.\Delta_{2}-\Delta_{1}=\arccos (3 / 5)\right]$ and/or $\Re\left[\exp \left[j\left(\Delta_{1}+\Delta_{2}\right)\right] \varphi_{\Phi}(-2)\right]=0$. Given the expression of $T_{1}$ in (12), $\arg \left(T_{1}\right)=\Delta_{1}+\Delta_{2}$, so that the condition (6) for non-isotropic $\mathrm{V}$ shaped arrays translates into

$$
\mathrm{E}[\cos (2 \Phi)] \cos \left(\Delta_{1}+\Delta_{2}\right)+\mathrm{E}[\sin (2 \Phi)] \sin \left(\Delta_{1}+\Delta_{2}\right)=0 .
$$

The performance measure (7), then equal to $\bar{C}=\frac{3}{2 \pi^{2}} \frac{5-3 \cos \left(\Delta_{1}-\Delta_{2}\right)}{\sin ^{2}\left(\Delta_{2}-\Delta_{1}\right)}$, reaches a minimum when $\Delta_{2}-$ $\Delta_{1}=\arccos (1 / 3)$, i.e., $\Delta_{2}-\Delta_{1}=70.52^{\circ}$. This optimal $\mathrm{V}$ array is not isotropic and achieves

$$
\bar{C}=\frac{27}{4 \pi^{2}}=0.6839 \text {. }
$$

This is significantly lower than for the isotropic $\mathrm{V}$ array (for which $\bar{C}$ equals $76 \%$ ) and quite close to optimal arrays obtained in Sec. III-A (for which $\bar{C}$ approaches $64 \%$ ).

\section{B. Prioritized Approach}

We study the following two functions $r_{1}\left(\Delta_{1}, \Delta_{2}\right)$ and $r_{-1}\left(\Delta_{1}, \Delta_{2}\right)$ corresponding to the right-hand side of (13) and (14), i.e. the normalized ECRBs on azimuth and elevation, respectively,

$$
r_{\epsilon}\left(\Delta_{1}, \Delta_{2}\right) \hat{=} \frac{3}{2 \pi^{2}} \frac{5-3 \cos \left(\Delta_{1}-\Delta_{2}\right)+\epsilon\left[5 \cos \left(\Delta_{1}-\Delta_{2}\right)-3\right] \Re\left[\exp \left[j\left(\Delta_{1}+\Delta_{2}\right)\right] \varphi_{\Phi}(-2)\right]}{\sin ^{2}\left(\Delta_{2}-\Delta_{1}\right)} .
$$

We set $\epsilon=1$ (resp. -1 ) if we are interested in minimizing the ECRB (normalized w.rt. the UCA) on the azimuth angle $\Phi$ (resp. elevation angle $\Theta$ ). We also define

$$
\alpha \hat{=}\left|\varphi_{\Phi}(2)\right| .
$$

We prove that the minimum of $r_{\epsilon}\left(\Delta_{1}, \Delta_{2}\right)$ is given by

$$
\begin{aligned}
\min _{\Delta_{1}, \Delta_{2}} r_{\epsilon}\left(\Delta_{1}, \Delta_{2}\right) & =\frac{3}{4 \pi^{2}} \frac{(3-5 \alpha)^{2}}{5-3 \alpha-4 \sqrt{1-\alpha^{2}}}, \text { if } \alpha \neq \frac{3}{5} \\
& =\frac{24}{5 \pi^{2}}, \text { if } \alpha=\frac{3}{5} .
\end{aligned}
$$


It is met at $r_{\epsilon}\left(\Delta_{1}^{\dagger}, \Delta_{2}^{\dagger}\right)=\min _{\Delta_{1}, \Delta_{2}} r_{\epsilon}\left(\Delta_{1}, \Delta_{2}\right)$ iff the following three conditions are satisfied

$$
\begin{aligned}
\cos \left(\Delta_{1}^{\dagger}-\Delta_{2}^{\dagger}\right) & =\frac{4 \sqrt{1-\alpha^{2}}+3 \alpha-5}{5 \alpha-3} \\
\cos \left(\Delta_{1}^{\dagger}+\Delta_{2}^{\dagger}\right) & =\epsilon \frac{\mathrm{E}[\cos (2 \Phi)]}{\alpha} \\
\sin \left(\Delta_{1}^{\dagger}+\Delta_{2}^{\dagger}\right) & =\epsilon \frac{\mathrm{E}[\sin (2 \Phi)]}{\alpha} .
\end{aligned}
$$

At the same time, the other cost function takes on

$$
r_{-\epsilon}\left(\Delta_{1}^{\dagger}, \Delta_{2}^{\dagger}\right)=\frac{3}{16 \pi^{2}} \frac{5 \alpha-3}{\sqrt{1-\alpha^{2}}}\left(5 \alpha+3+\frac{(5+3 \alpha)(3-5 \alpha)}{3 \alpha-5+4 \sqrt{1-\alpha^{2}}}\right)
$$

Notice that conditions (20)-(22) do not define a unique $\mathrm{V}$ array. For instance, if we let $\Delta_{3} \hat{=} \arccos \left[\left(4 \sqrt{1-\alpha^{2}}+3 \alpha-5\right) /(5 \alpha-3)\right]$ and $\Delta_{4}$ be the angle in $[0,2 \pi)$ whose cosine and sine are given by the right-hand side in (21)-(22), respectively; and if we define $\Delta_{5} \hat{=}\left(\Delta_{4}-\Delta_{3}\right) / 2$ and $\Delta_{6} \hat{=}\left(\Delta_{3}+\Delta_{4}\right) / 2$, then, we can prove that there are, in fact, two distinct $\mathrm{V}$ arrays solutions of our optimization problem. They are given by $\left(\Delta_{1}, \Delta_{2}\right)$ equal to $\left(\Delta_{5}, \Delta_{6}\right)$ and $\left(\Delta_{5}+\pi, \Delta_{6}+\pi\right)$.

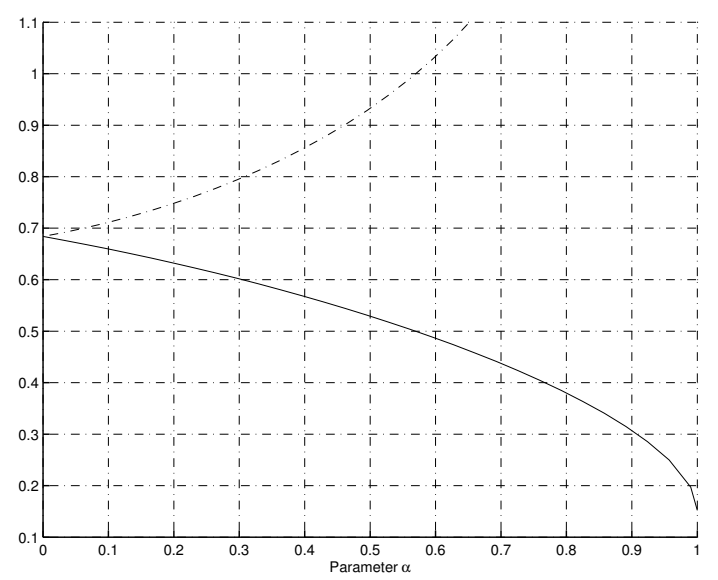

Fig. 2. Normalized ECRBs achieved by the optimal V array, as a function of parameter $\alpha$. Solid (dash-dotted) line refers to the normalized ECRB for the angle of interest (resp. on the other angle), i.e. (18) [resp. (23)].

We, first, study (18) that expresses the minimum normalized ECRB achievable by a V array, relative to our parameter of interest (azimuth $\Phi$ if $\epsilon=1$ or elevation $\Theta$ if $\epsilon=-1$ ). This minimum normalized ECRB is actually independent of the parameter of interest. It is presented by the solid line in Fig. 2 and is observed to decrease with $\alpha$, ranging from $68 \%$ for $\alpha=0$ (the unprioritized approach in Sec. IV-A) to $15.2 \%$ for $\alpha=1$ (the case of a deterministic prior, for example). 
The $\mathrm{V}$ array that minimizes the normalized ECRB for the DOA angle of interest does not minimize the normalized ECRB for the other DOA angle, except when $\alpha=0$, i.e., when array and source fit the condition (5) of the unprioritized approach. The ECRB (normalized w.r.t. the UCA) on the DOA angle other than the angle of interest is presented by the dashed line in Fig. 2. Interestingly, it is inferior to 1 when $\alpha$ is less than 0.59 . For such a distribution, the minimization of the normalized ECRB for a given angle (say for example azimuth) is not obtained at the expense of the normalized ECRB for the other angle (here, the elevation) in the sense that the latter is always less than 1, i.e., not degraded w.r.t. the UCA. For the typical case of an azimuth distributed as a Gaussian random variable with standard deviation $\sigma, \varphi_{\Phi}(-2)=\exp \left(-2 \sigma^{2}\right)$ and this situation takes place for $\sigma$ larger then $58.85^{\circ}$.

The $\mathrm{V}$ array is characterized by its shape (the angle $\Delta_{2}-\Delta_{1}$ between the two branches) and its orientation (angle $\Delta_{1}$ ). The first parameter $\Delta_{2}-\Delta_{1}$ is the same, whether we intend to minimize the normalized azimuth ECRB or the normalized elevation ECRB, as a result of (20) being independent of $\epsilon$. For a fixed optimal $\mathrm{V}$ array (i.e., a fixed $\Delta_{2}-\Delta_{1}$ ), there are 4 possible optimal orientations (i.e., $\Delta_{1}$ ). Two of them (rotation one of the other by $180^{\circ}$ ) minimize the MSE of the azimuth angle. The other two (also, rotation one of the other by $180^{\circ}$ ) minimize the MSE of the elevation angle.

\section{CONCLUSion}

Using elements from Bayesian estimation theory, we propose CRB-based antenna array DOA estimation performance criteria that take into account the availability of some prior information about the source (azimuth) angle. We define array geometry optimization problems in order to achieve improvement w.r.t. the commonly used UCA, and solve them by exhaustive search. Optimum arrays are obtained that minimize the normalized ECRB following two alternative strategies: (i) the two DOA parameters (azimuth and elevation) are declared of equal importance and the same reduction is required for both or, instead, (ii) emphasis is put on one of the angles. The constraint (i) leads to (large-sized) arrays that reduce the ECRB by $36 \%$ compared to similarly sized and spaced UCA, and so regardless of the available DOA prior information. By relaxing this constraint, approach (ii) leads to arrays with much lower normalized ECRB for one of the DOA angles. In all circumstances, optimal antenna arrays obtained here significantly outperform arrays in [10] and outperform UCAs even further, from the MSE point-ofview. The attractiveness of this analysis is further increased by the fact that optimum performance can be closely approached by V shaped arrays whose shape, orientation and performance are given analytically. 


\section{REFERENCES}

[1] Ü. Baysal and R. L. Moses, “On the geometry of isotropic arrays,” IEEE Trans. Signal Process., vol. 51, no. 6, pp. 1469-1478, Jun. 2003.

[2] M. Hawkes and A. Nehorai, "Effects of sensor placement on acoustic vector-sensor array performance," IEEE J. Ocean. Eng., vol. 24, no. 1, pp. 33-40, Jan. 1999.

[3] B. Porat and B. Friedlander, "Analysis of the asymptotic relative efficiency of the MUSIC algorithm," IEEE Trans. Acoust., Speech, Signal Process., vol. 36, no. 4, pp. 532-544, Apr. 1988.

[4] Ü. Oktel and R. L. Moses, “A Bayesian approach to array geometry design,” IEEE Trans. Signal Process., vol. 53, no. 5, pp. 19191923, May 2005.

[5] P. J. Bevelacqua and C. A. Balanis, "Optimizing antenna array Geometry for interference suppression,” IEEE Trans. Antennas and Propag., vol. 55, no. 3, pp. 637-641, Mar. 2007.

[6] H. Gazzah and S. Marcos, "Cramer-Rao bounds for antenna array design,” IEEE Trans. Signal Process., vol. 54, no. 1, pp. 336-345, Jan. 2006.

[7] F. Athley, C. Engdahl and P. Sunnergren, "On radar detection and direction finding using sparse arrays," IEEE Trans. Aerosp. Electron. Syst., vol. 43, no. 4, pp. 1319-1333, Oct. 2007.

[8] L. C. Godara and A. Cantoni, "Uniqueness and linear independence of steering vectors in array space," J. Acoust. Soc. Amer., vol. 70, no. 2, pp. 467-475, Aug. 1981.

[9] H. Gazzah and K. Abed-Meraim, "Optimum Ambiguity-Free Directional and Omni-Directional Planar Antenna Arrays for DOA Estimation,” IEEE Trans. Signal Processing, vol. 57, no. 10, pp. 3942-3253, Oct. 2009.

[10] H. Gazzah, "Optimum Antenna Arrays for Isotropic Direction Finding," IEEE Trans. Aerosp. Electron. Syst., vol. 47, no. 2, pp. 1482-1489, April 2011.

[11] A. J. Weiss, "On the accuracy of a cellular location system based on RSS measurements," IEEE Trans. Veh. Technol., vol. 52, no. 6, pp. 15081518, Nov. 2003.

[12] T. Filik and T. E. Tuncer, "Uniform and nonuniform V-shaped isotropic planar arrays," in Proc. IEEE Sensor Array and Multichannel Signal Processing Workshop, 2008, pp. 99-103.

[13] H. L. Van Trees and K. L. Bell, Bayesian Bounds for Parameter Estimation and Nonlinear Filtering/Tracking, H. L. Van Trees and K. L. Bell, Ed. John Wiley \& Sons, 2007.

[14] H. Gazzah and J.-P. Delmas, "Optimization of the antenna array geometry based on a bayesian DOA estimation criterion," in Proc. Int. Conf. Acoustics, Speech, Signal Processing (ICASSP), 2011, pp. 2544-2547.

[15] J. T.-H. Lo and S. L. Marple, Jr., "Observability conditions for multiple signal direction finding and array sensor localization,” IEEE Trans. Signal Processing, vol. 40, pp. 2641-2650, Nov 1992.

[16] K.-C. Tan, S. S. Goh and E.-C. Tan, "A study of the rank-ambiguity issues in direction-of-arrival estimation,” IEEE Trans. Signal Processing, vol. 44, pp. 880-887, Apr. 1996.

[17] D. Qi and X. Xianci, "DOA ambiguity vs. array configuration for subspace-based DF methods," in Proc. CIE International Conference of Radar, 1996, pp. 488-492.

[18] M. Gavish and A. J. Weiss, “Array geometry for ambiguity resolution in direction finding,” IEEE Trans. Antennas Propagat., vol. 39, pp. 143-146, Feb. 1991. 